\title{
Neutrino Cosmology and baryon asymmetry
}

\section{Abstract}

Pedro Cunha de Holanda (PQ), Anderson Luiz Brandão de Souza (IC)

One of the main open problems in modern cosmology is to understand the asymmetry between matter and antimatter from a state of equilibrium, called baryon asymmetry described by the Baryogenesis model. This research presents an extension of the Standard Model, in which Leptogenesis (asymmetry among the leptons) is a natural consequence of mass generation mechanism of Majorana neutrinos. The next step is to analyze as there is a possible transfer of asymmetry from leptons to baryons.

Key words: Majorana neutrinos, See-saw mechanism, Leptogenesis.

\section{Introduction}

In the original Standard Model of Particle Physics (SM), neutrinos are Dirac particles (spin 1/2 fermions) massless and single chirality, the lefthanded. The Dirac masses could be incorporated into the SM without many difficulties: through spontaneous symmetry breaking and adding the right-handed part for the Yukawa coupling with the Higgs boson, similarly as is done in the quarks sector [1]. However, neutrinos are the only particles Dirac, which do not have charge, can also be particles Majorana: massive fermions are their own antiparticle; which would be an extension of the SM. The objective of this study is to explore that possibility to try to explain some questions of Cosmology and Particle Physics still open: the mass generation of neutrinos and the Baryogenesis eventually transmitted by the Leptogenesis.

\section{Results and Discussion}

In the most general case, consider a spectrum comprising $n$ active neutrinos (which form lefthanded doublets with charged leptons, maintaining the symmetry $S U$ (2) $L \times U$ (1) $Y$ of the $\mathrm{SM}$ ) and $k$ sterile neutrinos (singlets right-handed, beyond on the SM). The mass Majorana is generated by spontaneous breaking the electroweak symmetry of the Weinberg coupling, although this is not renormalizable, it is understood that SM is an effective low-energy theory. The lagrangian mass term of this model is built on the basis formed by interaction eigenstates with the Higgs boson [2]:

$\begin{array}{ccccc}\mathcal{L}_{m} & =-\frac{1}{2} & {\left[\begin{array}{ll}\left(\nu_{L}^{\prime}\right)^{c} & \overline{\nu_{R}^{\prime}}\end{array}\right]} & {\left[\begin{array}{cc}0 & \left(M^{D}\right)^{T} \\ M^{D} & M^{R}\end{array}\right]} \\ \mathcal{L}_{m}=-\frac{1}{2} & \overline{N^{c}} & M & {\left[\begin{array}{c}\nu_{L}^{\prime} \\ \left(\nu_{R}^{\prime}\right)^{c}\end{array}\right]} & + \text { h.c. } \\ N & + \text { h.c. }\end{array}$

The interaction eigenstates have not well-defined mass, because the $M$ matrix is not diagonal on that basis. The vectors of the basis in which the matrix is diagonal are called mass eigenstates.

Note that, from the last expression, we conclude that the mass eigenstates are Majorana particles.
The existence of right-handed neutrinos is not expected for the SM and that model is valid only in the energy range below the electroweak unification, then here we make the assumption that the mass of singlets is much larger than the Dirac mass. Therefore, it effects a basis change so that mass matrix is diagonal block, decoupling light and heavy neutrinos,

$\tilde{M} \simeq\left(\begin{array}{cc}M_{\text {light }} & 0 \\ 0 & M_{\text {heavy }}\end{array}\right), \begin{aligned} & M_{\text {light }} \simeq-\left(M^{D}\right)^{T}\left(M^{R}\right)^{-1} M^{D} \\ & M_{\text {heavy }} \simeq M^{R}\end{aligned}$

This is the See-saw type I mechanism: active neutrinos are formed by linear combinations of the mass eigenstates of light neutrinos and sterile by heavy neutrinos; and the larger the sterile mass, the smaller the active mass, like a balance.

\section{Conclusions}

The extension of the SM presented in this research is able explaining the reason for the mass of active neutrinos are so smaller $(\sim \mathrm{eV})$ that the mass of the charged leptons $(\sim \mathrm{MeV})$, since most of the mass of neutrinos is with sterile, which not interact. If neutrinos are Majorana particles, the existence of sterile neutrinos is necessary to assign mass to active neutrinos and to explain, for example, flavors oscillation phenomenon; besides being a natural candidate for non-baryon dark matter. Finally, the lagrangian mass term written in function only Majorana particles is not invariant under transformations $U(1)$ and the lack of such symmetry does not guarantee the conservation of lepton number. In this scenario, the Leptogenesis is a natural consequence of mass generation mechanism of Majorana neutrino.

\section{Acknowledgement}

\section{A JAPESP}

Novaes, S. Standard Model: An Introduction. ArXiv:hepph/0001283, 2000.

${ }^{2}$ Akhmedov, E. Majorana neutrinos and other Majorana particles: Theory and experiment. ArXiv: 1412.3320v1 , 2014. 